\title{
MD or DM? Modified dynamics at low accelerations vs dark matter
}

\author{
Mordehai Milgrom* \\ Weizmann Institute \\ E-mail: moti.milgromeweizmann.ac.il
}

The MOND paradigm posits a departure from standard Newtonian dynamics, and from General Relativity, in the limit of small accelerations. The resulting modified dynamics aim to account for the mass discrepancies in the universe without non-baryonic dark matter. I briefly review this paradigm with its basic tenets, and its underlying theories-nonrelativistic and relativisticincluding a novel, bimetric MOND gravity theory. I also comment on MOND's possible connection to, and origin in, the cosmological state of the universe at large. Some of its main predictions, achievements, and remaining desiderata are listed. I then succinctly pit MOND against the competing paradigm of standard dynamics with cold, dark matter.

Some of the complaints leveled at MOND are: (i) "MOND was designed to fit rotation curves; so no wonder it is so successful in predicting them". This is both incorrect and quibbling: The first ever MOND rotation curve analysis was undertaken more then four years after the advent of MOND. And, even if MOND, epitomized by a very simple formula, could have been designed to predict hundreds of rotation curves, it would still be a great achievement. (ii) "MOND outperforms CDM only on small, galactic scales, where formation physics is anyhow very messy, but falls behind in accounting for 'simpler', large-scale phenomena". Quite contrarily, all the salient MOND predictions on galactic scales follow as unavoidable, simple, and immediate corollaries of the theory-independent of any messy formation scenario-just as Kepler's laws, obeyed by all planetary systems, follow from an underlying theory, not from complex formation scenarios. To think, as dark-matter advocates say they do, that the universal MOND regularities exhibited by galaxies will one day be shown to somehow follow from complex formation processes, is, to my mind, a delusion. What is left for MOND to explain on large scales is a little in comparison, and has to await a full fledged relativistic MOND theory. (iii) "The 'bullet cluster' shows that MOND still requires some matter that is dark". Yes, it has long been known that MOND does not fully remove the mass discrepancy in the cores of galaxy clusters. Some additional still-dark matter is needed. But this need not be THE "dark matter"; a small amount of the still-missing baryons, in some dark form (dead stars? cold gas clouds?), or perhaps (sterile?) neutrinos, could fit the bill.

Quarks, Strings and the Cosmos - Héctor Rubinstein Memorial Symposium

August 9-11 2010

AlbaNova, Stockholm, Sweden

${ }^{*}$ Speaker. 


\section{MOND introduced}

MOND, or modified dynamics, was propounded in the early 1980s [1], as a new theory of dynamics that accounts for the mass discrepancies in galactic systems without non-baryonic dark matter (DM). MOND hinges on the fact that the typical accelerations in such systems are many orders of magnitude smaller than those encountered in the solar system. It may be viewed, in some sense, as a modification of standard dynamics at "low accelerations". Some reviews of MOND can be found in [2, 3, 4, 5]. A historical account of DM and the MOND paradigms can be found in [6].

I start with some basic axioms for the new dynamics, such axioms as was the constancy of the speed light in special relativity, the equivalence of acceleration and gravity in General Relativity (GR), or the parceling of the radiation field in quantum theory. The reason for starting with such axioms, and not concentrating on a specific theory right from the outset, is twofold: 1 . We are not yet in the state where we have one universally accepted theory for MOND, even in the nonrelativistic (NR) regime. So, a set of basic principles from which to start can serve as a useful anchor and guide. 2. It turns out that there exists, in fact, a set of simply formulated basic tenets, from which alone follow a large number of major predictions, encompassing most of the basic, NR MOND phenomenology. These predictions are thus shared by all theories built on the axioms, which is a very useful knowledge.

The basic tenets of MOND, as they apply to NR matter systems governed by gravity, such as the solar system or galactic systems, are: (i) Admit into physics a new constant, $a_{0}$, with the dimensions of acceleration. It defines the boundary between the applicability domain of the standard dynamics (from which it disappears) and the new dynamics (in the phenomenology of which $a_{0}$ appears extensively), qualitatively similar to how $c$ appears in relativity, or $\hbar$ in quantum physics. (ii) A "correspondence principle": a NR MOND theory has Newtonian dynamics as its formal limit when $a_{0} \rightarrow 0$. (iii) In the opposite, deep-MOND limit, formally defined by $a_{0} \rightarrow \infty$, while all masses $m_{i} \rightarrow 0$ (or $G \rightarrow 0$ ), such that $m_{i} a_{0}$ (or $G a_{0}$ ) remain fixed, we require the theory to become scale invariant: dynamical evolution is invariant under $(t, \mathbf{r}) \rightarrow \lambda(t, \mathbf{r})$.

In more general contexts-such as in the relativistic regime, or when non-gravitational interactions are involved-we may have to generalize the above basic tenets. For example, we shall see that there are relativistic MOND theories that do not tend exactly to GR in the limit $a_{0} \rightarrow 0$ (while their NR limit does tend to Newtonian gravity in the limit).

\section{MOND theories}

Here, I discuss briefly theories that incorporate the basic tenets of MOND. In this connection, note the numerical coincidence between the value of $a_{0} \approx 10^{-8} \mathrm{~cm} \mathrm{~s}^{-2}$, as determined from numerous phenomena in which it appears (see below), and the values of some cosmological acceleration parameters, as has been noted and elaborated on many times along MOND's history (e.g. in $[1,7,8,9,10,11])$. We have

$$
\bar{a}_{0} \equiv 2 \pi a_{0} \approx c H_{0} \approx(\Lambda / 3)^{1 / 2}
$$

where $H_{0}$ is the Hubble constant, and $\Lambda$ the cosmological constant (CC) as now deduced from various cosmological data. This striking proximity, if it is more than a mere coincidence, may be 
an important hint in constructing MOND theories. It would be desirable (if not necessary) that a MOND theory should account for this numerical proximity.

Quantum theory and relativity depart from Newtonian dynamics, in the first place, by introducing novel world pictures. At the more "technical" level, these novel concepts then form the basis for specific theories described by this or that action. There are intriguing hints that MOND also rests on some novel world picture (e.g. [10, 12]). However, so far, the only palpable and "practical" progress made has been in constructing modified actions for MOND theories, based on the standard concepts of Newtonian dynamics and GR. These theories invariably involve some function, put in by hand, that interpolates between the MOND and the neoclassical (pre-MOND) regime. This, to my mind, testifies strongly to the "effective" nature of these theories.

The more tractable approach for building MOND theories has, so far, proven to be modifying only the field equation for the gravitational potential (the Poisson equation), leaving Newton's law of inertia intact. In the relativistic context, the equivalent approach is to adhere to metric theories, keep the standard matter action of GR, with the standard (minimal) matter coupling to the metric, but modify the equations that determine the metric from its matter sources. I call such theories modified-gravity (MG) theories.

Efforts in this direction have already yielded some NR theories, and three classes of relativistic MOND theories (TeVeS, MOND adaptations of Einstein-Aether theories, and BIMOND, to be described below).

Other theories, loosely called "modified inertia" (MI) theories, involve modifying Newton's second law, or generally, modifying the matter actions (both NR and relativistic). This approach has proven more difficult to implement, and remains relatively unexplored. It seems to me, however, to be an attractive avenue, worth pursuing; it is a natural framework for connecting MOND with cosmology, as perhaps pointed to by the above coincidence. Some more detailed results on this approach can be found in [8, 10, 13].

The new physics entailed by MOND shows up in full force only in galactic systems, whose dynamics is governed by gravity. It is thus difficult to distinguish observationally between the two classes. Important differences in the predictions of the two classes of theories exist even for purely gravitational systems, but they have, so far, not been brought to bear on the question of which of the two approaches is phenomenologically superior to the other.

I now describe briefly some of the MOND theories propounded to date.

\subsection{Nonrelativistic theories}

\subsubsection{Nonlinear Poisson equation for the gravitational potential $\phi$}

For a gravitating system made of point-like, individually-test masses, whose trajectories are $\mathbf{r}_{i}(t)$, we have [14]

$$
\ddot{\mathbf{r}}_{i}=-\vec{\nabla} \phi\left(\mathbf{r}_{i}\right), \quad \vec{\nabla} \cdot\left[\mu\left(|\vec{\nabla} \phi| / a_{0}\right) \vec{\nabla} \phi\right]=4 \pi G \rho,
$$

derived from a generalized Poisson action. In the limit $a_{0} \rightarrow 0: \mu(x \rightarrow \infty) \rightarrow 1$. In the deep-MOND limit ( $a_{0} \rightarrow \infty, a_{0} G$ constant): the first eq.(2.2) implies that $\phi$ has zero dimensions under scaling, and thus for the second eq.(2.2) to give scale invariance we have to have

$$
\mu(x \ll 1) \propto x,
$$


and we normalize $a_{0}$ so equality holds. The nonlinear Poisson equation for the gravitational potential $\phi$, thus reduces to the 3 -harmonic Poisson equation $\vec{\nabla} \cdot(|\vec{\nabla} \phi| \vec{\nabla} \phi)=4 \pi G a_{0} \rho$.

The theory then becomes invariant to conformal transformations in space, with many applications and possible matter-of-principle ramifications [15, 12].

\subsubsection{Quasilinear formulation (QUMOND)}

The field equations for the gravitational potential (derived from an action) are here [16]

$$
\Delta \phi=\vec{\nabla} \cdot\left[v\left(\left|\vec{\nabla} \phi^{*}\right| / a_{0}\right) \vec{\nabla} \phi^{*}\right], \quad \text { where } \quad \Delta \phi^{*}=4 \pi G \rho .
$$

This requires solving only a linear Poisson equation (twice), and is rather easier to solve than the nonlinear Poisson equation. Scale invariance in the deep-MOND limit dictates $y^{1 / 2} v(y \rightarrow 0) \rightarrow$ const. (again $a_{0}$ normalized so that the limit is 1 ).

\subsection{3 "Modified inertia"}

In [8], I considered (NR) action-based theories, whereby the gravitational potential is still determined from the Poisson equation, but the particle equation of motion is of the form

$$
\mathbf{A}\left[\{\mathbf{r}(t)\}, a_{0}\right]=-\vec{\nabla} \phi
$$

(replacing $\ddot{\mathbf{r}}=-\vec{\nabla} \phi$ ), where $\mathbf{A}$ is a functional of the whole trajectory $\{\mathbf{r}(t)\}$, with the dimensions of acceleration. For $a_{0} \rightarrow 0, \mathbf{A} \rightarrow \ddot{\mathbf{r}}$. Here, $\phi$ has dimension -1 under scaling (from the Poisson equation); so, to have scale invariance in the deep-MOND limit, we have to have

$$
\mathbf{A}\left[\{\mathbf{r}(t)\}, a_{0}\right] \rightarrow a_{0}^{-1} \mathbf{Q}(\{\mathbf{r}(t)\})
$$

where $\mathbf{Q}$ has dimensions of acceleration squared.

I showed that if such an equation of motion is to follow from an action principle, enjoy Galilei invariance, and have the above Newtonian and MOND limits, it has to be time nonlocal. ${ }^{1}$ Such nonlocal theories (which also have to be nonlinear-an inherent property of MOND theories) are not easy to construct. We do not yet have a fully acceptable theory in this vein, even in the NR regime. Only some toy theories have been partly explored [8, 10, 13]. In particular, in [10], I discussed a heuristic idea, whereby MOND, indeed inertia itself, can result from an effect of the vacuum, and where the origin of $a_{0}$ in cosmology also emerges, as per eq.(2.1). The vacuum then serves as an absolute inertial frame (acceleration with respect to the vacuum is detectable, e.g., through the Unruh effect).

There is an important and robust prediction shared by all theories in the class: For circular trajectories in an axisymmetric potential, eq.(2.5) has to take the form

$$
\mu\left(V^{2} / R a_{0}\right) V^{2} / R=-d \phi / d R,
$$

where, $V$ and $R$ are the orbital speed and radius, and $\mu(x)$ is universal for the theory, and is derived from the expression of the action specialized to circular trajectories; $\mu(x \ll 1) \approx x, \mu(x \gg 1) \approx 1$.

\footnotetext{
${ }^{1}$ An interesting possibility, which I have explored only superficially, is of a local theory that enjoys a symmetry other than Galilei, which reduces to Galilei invariance for $a_{0} \rightarrow 0$.
} 
Thus even without specifying the theory, this class makes a very useful prediction for galaxy rotation curves (RC). It is, indeed, eq.(2.7)-which gives the MOND RC in simple terms of the Newtonian curve-that has been used in all MOND RC analyses to date. The predictions of modifiedgravity theories are less straightforward to compute, and require a computation of the whole gravitational field for each galaxy anew.

\subsection{Relativistic theories}

\subsubsection{TeVeS}

The Tensor-Vector-Scalar (TeVeS), relativistic formulation of MOND, has been put forth by Bekenstein [17], building on ideas by Sanders [18]. The theory has been discussed and reviewed extensively (e.g., recently, in [19, 20]); so I describe it very briefly.

Gravity is carried by a metric $g_{\alpha \beta}$, a vector field $\mathscr{U}_{\alpha}$, and a scalar field $\phi$, while matter degrees of freedom couple minimally to the "physical" metric $\tilde{g}_{\alpha \beta}=e^{-2 \phi}\left(g_{\alpha \beta}+\mathscr{U}_{\alpha} \mathscr{U}_{\beta}\right)-e^{2 \phi} \mathscr{U}_{\alpha} \mathscr{U}_{\beta}$.

TeVeS reproduces MOND phenomenology for galactic systems in the NR limit, with a certain combination of its constants playing the role of $a_{0}$. In particular, when $a_{0} \rightarrow 0$, the NR limit goes to Newtonian gravity. However, the relativistic theory does not go exactly to GR in the same limit. This departure of TeVeS from GR even for very high accelerations might leave observable effects in the solar system (see, e.g., [21]).

As in GR, the potential that appears in the expression for lensing by NR masses (such as galactic systems) is the same as that which governs the motion of massive particles.

Cosmology, the CMB, and structure formation in TeVeS have been considered in [22, 23, 24, 25, 26]. It was shown that there are elements in TeVeS that could mimic cosmological DM, although no fully satisfactory application of TeVeS to cosmology has been demonstrated.

Gravitational waves in TeVeS have been considered in [17, 27].

\subsubsection{MOND versions of Einstein-Aether theories}

Zlosnik et al. [28] have proposed and discussed MOND adaptations of Einstein-Aether (EA) theories. In EA theories (e.g., [29], and references therein), gravity is carried by a metric, $g_{\mu \nu}$, as well as a vector field, $A^{\alpha}$. To the standard Einstein-Hilbert Lagrangian for the metric one adds the terms

where

$$
\mathscr{L}(A, g)=\frac{a_{0}^{2}}{16 \pi G} \mathscr{F}(\mathscr{K})+\mathscr{L}_{L}
$$

$$
\begin{gathered}
\mathscr{K}=a_{0}^{-2} \mathscr{K}_{\gamma \sigma}^{\alpha \beta} A_{; \alpha}^{\gamma} A_{; \beta}^{\sigma} . \\
\mathscr{K}_{\gamma \sigma}^{\alpha \beta}=c_{1} g^{\alpha \beta} g_{\gamma \sigma}+c_{2} \delta_{\gamma}^{\alpha} \delta_{\sigma}^{\beta}+c_{3} \delta_{\sigma}^{\alpha} \delta_{\gamma}^{\beta}+c_{4} A^{\alpha} A^{\beta} g_{\gamma \sigma},
\end{gathered}
$$

and $\mathscr{L}_{L}$ is a Lagrange multiplier term that forces the vector to be of unit length. The asymptotic behavior of $\mathscr{F}$, at small and large arguments, give the deep-MOND behavior and GR, respectively.

\subsubsection{Bimetric MOND gravity}

Bimetric MOND gravity (BIMOND) [30, 31, 32] is a class of relativistic theories governed by the action

$$
I=-\frac{1}{16 \pi G} \int\left[\beta g^{1 / 2} R+\alpha \hat{g}^{1 / 2} \hat{R}-2(g \hat{g})^{1 / 4} a_{0}^{2} \mathscr{M}\right] d^{4} x+I_{M}\left(g_{\mu v}, \psi_{i}\right)+\hat{I}_{M}\left(\hat{g}_{\mu v}, \hat{\psi}_{i}\right) .
$$


It involves two metrics, $g_{\mu \nu}$ and $\hat{g}_{\mu \nu}$, whose Ricci scalars are $R$ and $\hat{R}(c=1) . \mathscr{M}$ is a dimensionless, scalar function of the two metrics and their first derivatives.

The novelty in BIMOND-compared with earlier bimetric theories, much discussed since the early 1970s-is in the choice of the interaction term. The difference of the two Levi-Civita connections

$$
C_{\beta \gamma}^{\alpha}=\Gamma_{\beta \gamma}^{\alpha}-\hat{\Gamma}_{\beta \gamma}^{\alpha}
$$

is a tensor that acts like the relative gravitational accelerations of the two sectors. This is particularly germane in the context of MOND, where, with $a_{0}$ at our disposal, we can construct from $a_{0}^{-1} C_{\beta \gamma}^{\alpha}$ dimensionless scalars to serve as variables on which $\mathscr{M}$ depends. In particular, the scalars constructed from the quadratic tensor

$$
\Upsilon_{\mu \nu} \equiv C_{\mu \lambda}^{\gamma} C_{v \gamma}^{\lambda}-C_{\mu \nu}^{\gamma} C_{\lambda \gamma}^{\lambda}
$$

have particular appeal; e.g.,

$$
\Upsilon=g^{\mu v} \Upsilon_{\mu v}, \quad \hat{\Upsilon}=\hat{g}^{\mu v} \Upsilon_{\mu \nu} .
$$

The terms $I_{M}$ and $\hat{I}_{M}$ are the matter actions for standard matter and for putative twin matter (TM), whose existence is suggested (but not required) by the double metric nature of the theory. Matter degrees of freedom $\psi_{i}$ couple only to the standard metric $g_{\mu \nu}$, while TM couples only to $\hat{g}_{\mu \nu}$. In matter-TM symmetric versions of the theory, assumed below for concreteness, we have $\alpha=\beta$, and $g_{\mu \nu}, \hat{g}_{\mu \nu}$ appear symmetrically in $\mathscr{M}$. There are good reasons to take $\beta \approx 1$.

Matter equations of motion in the two sectors, are the standard ones, each with its own metric.

The gravitational field equations are

$$
\beta G_{\mu v}+S_{\mu v}=-8 \pi G \mathscr{T}_{\mu \nu}, \quad \beta \hat{G}_{\mu v}+\hat{S}_{\mu v}=-8 \pi G \hat{\mathscr{T}}_{\mu \nu},
$$

with $G_{\mu \nu}, \hat{G}_{\mu \nu}$ the respective Einstein tensors, and $\mathscr{T}_{\mu \nu}, \hat{\mathscr{T}}_{\mu \nu}$ the energy-momentum tensors for the two sectors.

The tensors $S_{\mu v}$ and $\hat{S}_{\mu \nu}$ have the schematic forms

$$
S_{\mu v}=\left(Q\{C\}_{\mu \nu}^{\lambda}\right)_{; \lambda}+N\left\{C^{2}\right\}_{\mu v}+a_{0}^{2} P g_{\mu \nu}, \quad \hat{S}_{\mu v}=\left(\hat{Q}\{C\}_{\mu \nu}^{\lambda}\right)_{: \lambda}+\hat{N}\left\{C^{2}\right\}_{\mu v}+a_{0}^{2} \hat{P} \hat{g}_{\mu v}
$$

where $\{C\}$ are tensors linear, and $\left\{C^{2}\right\}$ quadratic, in $C_{\mu \nu}^{\lambda}$.

Here are, briefly, some consequences expanded on in [30, 31, 32], assuming $\beta=\alpha=1$.

Imposing $\mathscr{M}^{\prime}(z \rightarrow \infty) \rightarrow 0$, the limit of the theory for $a_{0} \rightarrow 0$, is two uncoupled copies of GR with a $\mathrm{CC}, \Lambda \sim a_{0}^{2} \mathscr{M}(\infty)$.

For the choice of scalar arguments as in eq.(2.13), we get in the NR limit $g_{\mu v} \approx \eta_{\mu v}-2 \phi \delta_{\mu v}$, as in GR, but here $\phi$ is determined from a NR MOND theory. Thus, massive test particles and photons "see" the same potential.

BIMOND, with its two metrics, naturally accommodates TM. Matter and TM do not interact at all in the high-acceleration regime, and repel each other in the MOND regime [31]: In the deepMOND regime, TM behaves as having a gravitational mass opposite in sign to that of matter. This fits naturally with the (space) conformal invariance of the theory in this limit.

Exactly symmetric matter-TM systems obey GR with a CC, $\Lambda \sim a_{0}^{2} \mathscr{M}(0)$. 
Cosmology is preliminarily discussed in [33, 30, 32]: In an interesting special case, where the universe is matter-TM symmetric, there are no MOND effects, apart for the appearance of the $\mathrm{CC}, \sim a_{0}^{2}$. The "coincidence" (2.1) thus follows naturally in BIMOND. In this case cosmology is the standard FRW one, with a CC, retaining the successes of early-universe cosmology, such as nucleosynthesis. Other MOND effects, cosmological and local, only appear with matter-TM separation (or if there is no TM). The expected CMB fluctuations in BIMOND have not been studied yet.

Structure formation: some aspects, relevant to weak-inhomogeneities, are discussed in [32]. Even in a symmetric universe, if the initial quantum fluctuations are not identical in the two sectors, matter and TM segregate efficiently, since density differences grow much faster that the sum. The inhomogeneities of the two matter types develop, eventually, into mutually avoiding, cosmic webs.

$S_{\mu \nu}$ in eqs.(2.14)(2.15) can act as the energy-momentum tensor of cosmological DM, beside its contribution to the $\mathrm{CC}$.

Note that the MOND limit occurs, in BIMOND, when the acceleration difference in the two sectors is small compared with $a_{0}$. This quantity nearly equals the matter acceleration alone, in pure-matter systems-such as presumably are the well scrutinized systems (stars, galaxies, etc.)-but not in well mixed matter-TM configurations.

\subsection{Theories with microscopic basis}

There are suggestions to obtain MOND phenomenology in various microscopic-physics scenarios. For example, an omnipresent, polarizable, dark-mater medium [11], an assumption of properly tailored baryon-DM interactions [34], the "dark fluid" approach [35], various versions of gravity as an entropic effect [36, 37, 38], vacuum effects [10], and Hořava gravity [39].

\section{MOND phenomenology}

\subsection{Kepler-like laws}

Clearly, each MOND theory has its own specific predictions regarding the dynamics of gravitating systems, such as planetary systems, galaxies, galactic systems, and the universe at large. It is possible, however, to distill from these a number of predicted, general laws. This is analogous to the extraction of Kepler's laws from Newtonian dynamics. What is more, many of these corollaries follow essentially only from the basic tenets of MOND, and are thus valid in all MOND theories. It is also noteworthy that these MOND laws are independent from the DM point of view, in the sense that one can construct, for example, model populations of baryons-plus-DM galaxies that satisfy any subset of these corollaries, but not the others. This means that in the framework of the DM paradigm they would each require a separate explanation. Following are a few of these corollaries.

- Asymptotic independence of orbital velocities on the size of the orbit: Under space-time scaling, radii scale, but velocities do not, resulting in radius-independent orbital velocities in the MOND regime (large radii) around a point mass, $M$, (or around any mass well within the orbital radius). This fact underlies the prediction of asymptotically flat RCs of disc galaxies: $V(r) \rightarrow V_{\infty}$. See Figs. 2 and 3 . 


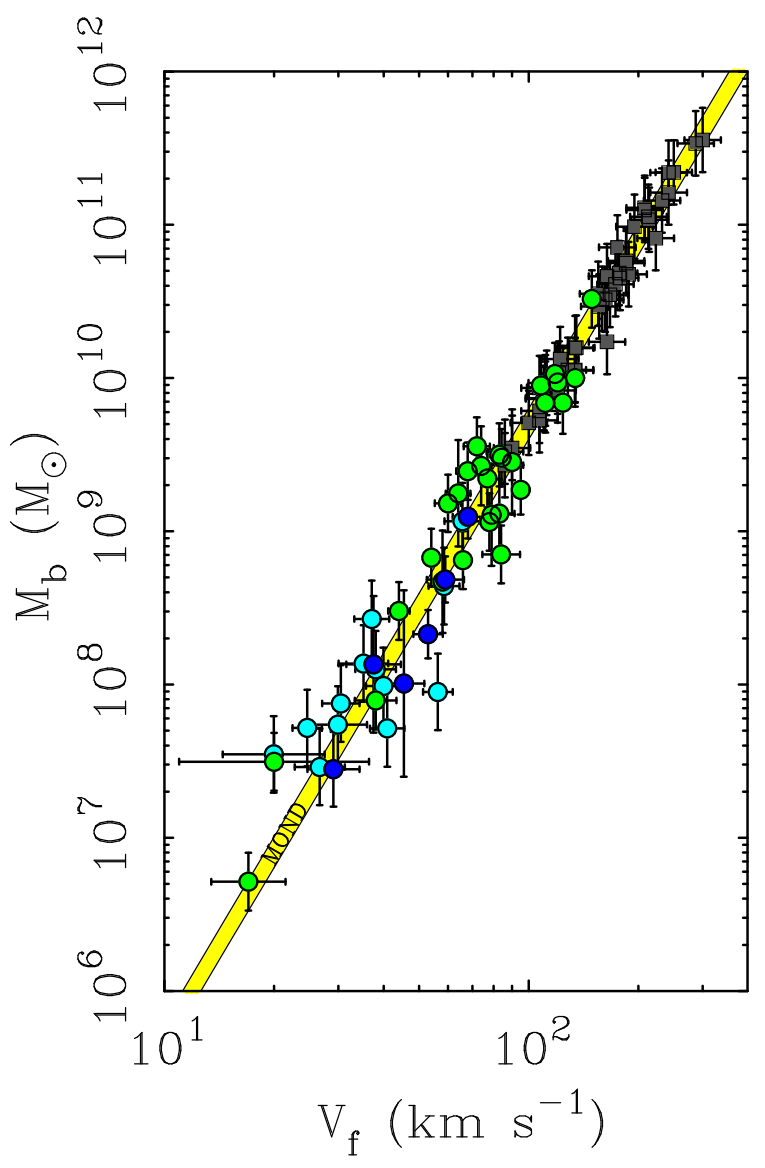

Figure 1: The total baryonic mass of disc galaxies plotted against the plateau (asymptotic) rotational speed. The (yellow) band is the MOND prediction (whose width represents the uncertainty in $a_{0}$ as determined by the data). From [40].

- The mass-asymptotic-velocity relation: With only $a_{0}$ available as a new dimensioned constant, we must have (equality, rather than proportionality, defines the normalization of $a_{0}$ )

$$
V_{\infty}^{4}=M G a_{0} .
$$

This prediction underlies the observed baryonic Tully-Fisher relation (see Fig. 1). The data is consistent with zero intrinsic scatter about the MOND prediction [40].

- In galaxies where $V^{2} / R>a_{0}$ in the inner parts, the mass discrepancy appears always around the radius where $V^{2} / R=a_{0}$, amply vindicated in many analyses.

- Isothermal spheres (IS)-approximating ellipticals and other spherical stellar systems-must have mean surface densities $\bar{\Sigma} \lesssim a_{0} / G$ : Newtonian ISs have, perforce, infinite mass. In MOND, they have a finite mass, but their mean acceleration has to be $\langle a\rangle \lesssim a_{0}$ to enjoy the MOND protection $(\langle a\rangle \sim G \bar{\Sigma}$ ). (Non-isothermal spheres, such as stars, can have arbitrarily high $\bar{\Sigma}$.) For comparison with the data see e.g., [41] 
- For pressure-supported systems, characterized by a velocity dispersion $\sigma$, the ratio $\eta=\frac{\sigma^{4}}{M G a_{0}}$ has to be of order unity, but depends somewhat on the definition of $\sigma$, on the degree of isotropy, on the proximity to the MOND regime, etc. This prediction underlays the observed Faber-Jackson relation between the luminosity and velocity dispersion of elliptical galaxies, and extends to globular clusters, galaxy clusters, etc. (e.g., [41]). Intrinsic scatter, or systematic deviation, is predicted because of possible scatter, or systematics, in $\eta$.

- The central surface density of "dark halos" is $\approx a_{0} / 2 \pi G[42,43,44]$.

- Added stability of discs with $\bar{\Sigma} \lesssim a_{0} / G$; i.e., discs whose bulk is in the MOND regime, where accelerations scale as $a \propto M^{1 / 2}$, instead of the Newtonian $a \propto M$. Thus $\delta a / a=(1 / 2) \delta M / M$, leading to a weaker response to perturbations [45].

- Disc galaxies have both a disc AND a spherical "DM" components [46].

- MOND theories are inherently nonlinear. This leads to a generic prediction of an "Externalfield effect" (EFE): The internal dynamics in a subsystem is affected by the acceleration with which it is falling in a mother system. MOND theories do not obey the strong equivalence principle, which prevents such effects in GR.

\subsection{Rotation curves of disc galaxies}

At present, full rotation curve (RC) analysis of disc galaxies, constitutes, arguably, the most comprehensive and robust manifestation of the mass discrepancy, and offers the best touchstone for testing alternative dynamics. Given the distribution of baryons in a galaxy, A MOND MG theory predicts the full gravitational field of the galaxy. In particular, it predicts the field in the midplane, which, in turn, determines the rotation curve. In MI theories, the gravitational field is the standard one, but particle trajectories are predicted by the specific theory. Even though we do not yet have a full-fledged MI MOND theory, as already mentioned, it can be shown that all such theories predict a simple, universal, algebraic relation between the MOND and Newtonian accelerations, on circular orbits, in an axisymmetric potential: relation (2.7); enough to predict the $\mathrm{RC}$ for a given galaxy. This relation was used in all MOND RC analyses to date. The exact choice of the universal function $\mu(x)$ appearing in eq.(2.7) turns out to affect only a little the predicted RCs [given that it satisfies the required asymptotic limits $\mu(x \ll 1) \approx x, \mu(x \gg 1) \approx 1]$. Small differences between the predictions of different forms of $\mu(x)$ for high-acceleration galaxies, do exist, of course, and are being explored. ${ }^{2}$ Some computations of RC predictions of MG theories have shown that these do not differ much from those of MI, although differences do exist, and can, in principle, be used to distinguish between the two classes of theories. ${ }^{3}$ Part of the reason that the predictions of the two classes of theories are similar, is that the general form of the RC is predicted by the basic MOND

\footnotetext{
${ }^{2}$ For the many low-acceleration galaxies that have been analyzed, where $a \ll a_{0}$ everywhere in the galaxy, only the $x \ll 1$ behavior of $\mu(x)$ enters.

${ }^{3}$ some of the differences may perhaps be compensated by adjusting the choice of the interpolating functions $\mu(x)$, which appears in both classes. Note that $\mu(x)$ appears in different ways in the two theory types. In existing MG theories $\mu$ appears in the gravitational action, and would enter all phenomena; in MI formulations, $\mu$ pertains only to circular orbits in axisymmetric potentials.
} 

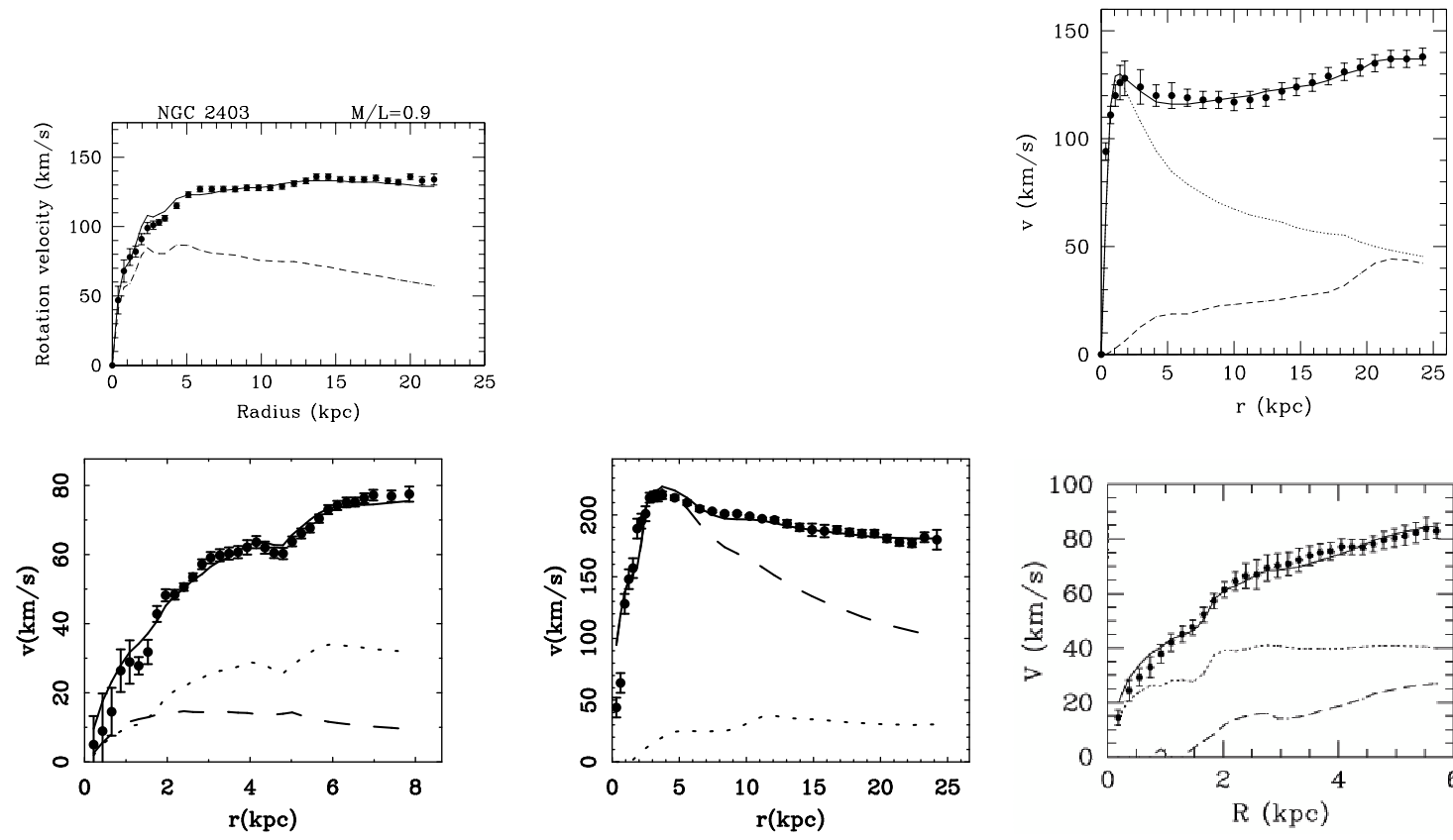

Figure 2: Rotation curves for galaxies across the gamut of RC types, from low-mass-low-speed galaxies that are everywhere of low-acceleration, to high-mass-high-speed galaxies that have high accelerations in the inner parts. The solid lines are the MOND curves; other lines are Newtonian contributions from the stars and gas (from [2], [47], and curtesy of Bob Sanders).

tenets and so is shared by all MOND theories. ${ }^{4}$ Additionally, for spherical systems, a relation of the form (2.7) is also exact for MG formulations.

Contrary to MOND, DM does not predict the RCs of individual galaxies from the baryon distribution. One can, at best, try to fit a DM halo, taken from a several-parameter family of DM halos predicted by this or that concept of DM (for example, the NFW-like halos predicted by CDM).

Figures. 2 and 3 show some MOND rotation curves for disc galaxies. In Fig. 2, I put together galaxies covering a large part of the range of galaxy types. Some of these show that the predicted MOND curves reproduce even local features on the observed RC. These features can be traced back to features in the baryon mass distribution of the galactic disc. They tend to wash out even in the best DM fits, since it is not expected that the same feature that appears in the disc-like baryon distribution, also appears in the spheroidal DM mass distribution.

\subsection{Elliptical galaxies}

The acceleration field around elliptical galaxies has to be probed by means other then RCs; these are generically less robust. There are, e.g., several strong-lensing studies, which probe the very inner parts; they are inconclusive, as the deduced mass discrepancies there are small and

\footnotetext{
${ }^{4}$ The asymptotic velocity is predicted to be constant and its value determined uniquely from the total mass, and, the velocity starts from zero at the center. In a high acceleration galaxy, the predicted velocity takes the Newtonian values roughly up to the radius, $R$, where $V(R)^{2} / R=a_{0}$, then the MOND behavior takes over.
} 

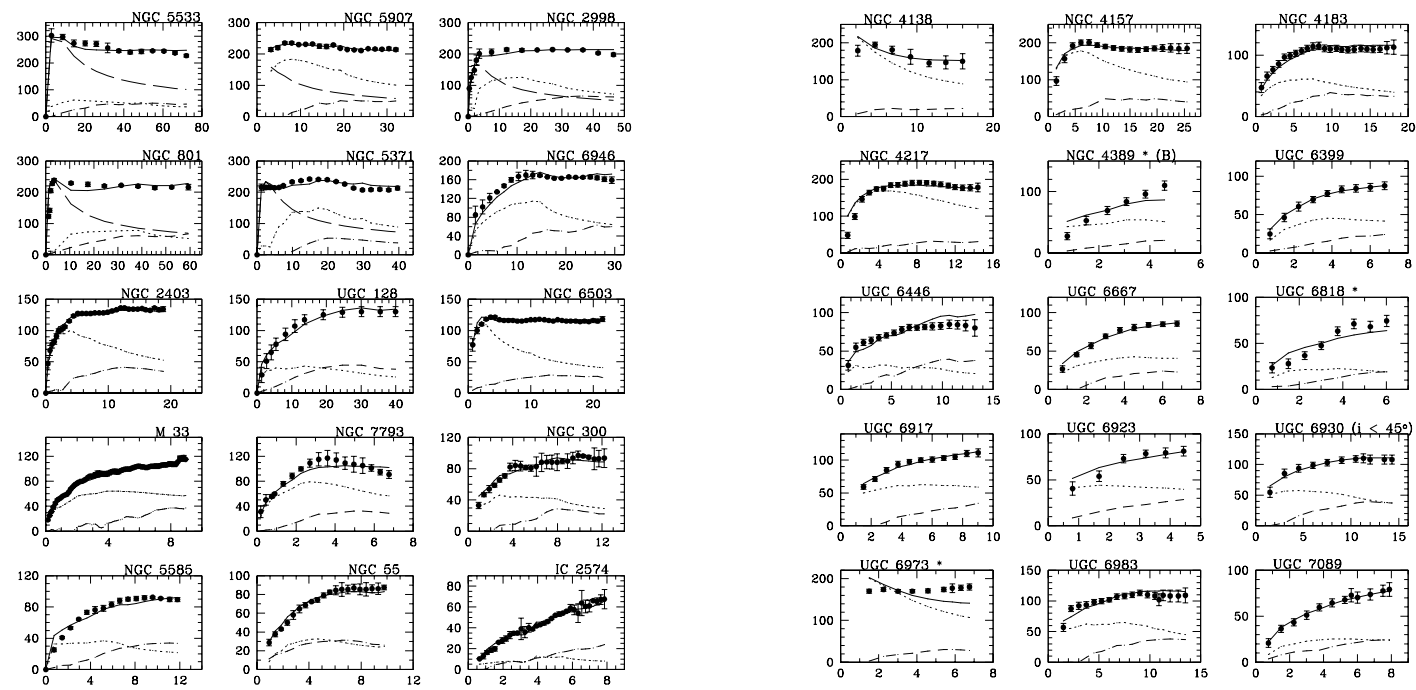

Figure 3: More MOND results for galaxy RCs (from [2]). The solid lines are the MOND curves; the other curves are based on Newtonian dynamics: for the stellar disc (dots), gas (dash dots), and stellar bulge (long dashed) contributions.

uncertain. Concentrating on studies that go to large galactic radii of isolated elliptical galaxies, I mention here the analyses of [48] and [49], who find good agreement with MOND prediction, in analyses based on planetary-nebulae, or companion-galaxy, probes. Recently, [50] published a mass analysis of the isolated elliptical NGC 720, based of x-ray-gas hydrostatics. They find that the mass discrepancy at a radius of $100 \mathrm{Kpc}$ is $\sim 10$. I note here that this is in very good agreement with the prediction of MOND: The MOND acceleration there is $g(100 \mathrm{Kpc}) / a_{0} \equiv x \approx 0.11$, and the predicted discrepancy is $1 / \mu(x)$, which is $\approx 1 / x$ for small $x$.

\subsection{Systems of galaxies}

MOND has been tested also on systems of galaxies, for example, on poor galaxy groups, in [51], and on super clusters; e.g., in [52]. These analyses showed that the very large mass discrepancies shown by these systems disappear in MOND, within the uncertainties.

However, analyses of galaxy clusters, employing x-ray-gas hydrostatics, and gravitational lensing, shows a persistent, remaining mass discrepancy, even when MOND is used (see e.g., [53. 54], and references therein, for earlier findings to this effect, going back to the early 1990s): The typical mass discrepancy of galaxy clusters within a few megaparces of the center, which by standard dynamics is of the order of a factor 10, is reduced in MOND to about a factor of two only. The deduced density distribution of the MOND "phantom" DM is similar to that of galaxies, and is rather more centrally concentrated than that of the x-ray gas, which makes the lion share of the baryons. As a result, the remaining mass discrepancy is rather more pronounced near the centers of the clusters. A more detailed discussion of this remaining discrepancy, and of possible explanations of it, are reviewed in [55], advocating, specifically, that the discrepancy is due to yet 
undetected baryons in clusters. Other suggestions involve neutrinos as the remaining cluster DM [56, 57]. The much bruited discrepancy shown by the colliding, double "bullet cluster", is exactly what is expected from previous analyses of single clusters, and does not further redound to the cluster conundrum in MOND.

\subsection{Cosmological "dark matter"}

Accounting fully for all the cosmological effects attributed conventionally to cosmological DM, is a remaining challenge for MOND. (The effects of "dark energy" can be naturally gotten in MOND theories, without "dark energy", as we saw above, e.g., in connection with BIMOND.) As has been amply shown, there are MOND effects that mimic some aspects of cosmological DM (e.g., the enhanced rate of the growth of perturbations, which occurs in MOND because gravity is augmented at low accelerations). But a full accounting may have to await the advent of a fundamental theory of MOND. I discuss this issue some more in the last section.

\section{4. $a_{0}$ and its significance}

As shown in sec. 3.1, $a_{0}$ appears in various laws predicted by MOND; its value is determined, consistently, by matching these predictions to the data. Thus, $a_{0}$, and the associated MOND phenomenology, is here to stay, no matter what the interpretation of MOND is. Arguably, the best constraints on $a_{0}$ come from the measured proportionality factor of the mass-asymptotic speed relation (3.1), and from more detailed rotation-curve analysis (for a recent account of such determinations, see [40]). As shown by eq.(2.1), the value found $a_{0}=(1.20 \pm 0.25) \times 10^{-8} \mathrm{~cm} \mathrm{~s}^{-2}$, is near some cosmologically significant accelerations. Is this a mere coincidence? Assuming that it is not, and that it does have a fundamental significance, several questions arise: Is the approximate equality $\bar{a}_{0} \approx c H_{0}$, where $H_{0}$ is today's Hubble constant, the more fundamental one, and the second equality is then just a result of the celebrated and mysterious "cosmic coincidence" $H_{0}^{2} \approx \Lambda / 3$ ? In this case, does this signify that $a_{0}$ varies with cosmic time in proportion to the expansion rate, so as to maintain the equality at all times? Then, antropic explanations of the cosmic coincidence suggest themselves ( [7, 9]). Is the second equality, $\bar{a}_{0} \approx c(\Lambda / 3)^{1 / 2}$, the more fundamental one? Is then $a_{0}$, as is purportedly $\Lambda$, a veritable constant? In either case, the information about the state of the universe at large, encoded in $H_{0}$ and/or $\Lambda$, somehow has to enter local dynamics in small systems, such as galaxies (as discussed, e.g., in [10]). Alternatively, we can have a scheme in which the $a_{0}$ appears in some underlying theory of MOND, and so it both fixes the CC, and also appears in local dynamics. This happens, e.g., in the theory discussed in [11], and in BIMOND [30].

The double equality (2.1) doubles the mystery of the cosmic coincidence: not only is the expansion rate today comparable with the (fixed?) value of $(\Lambda / 3)^{1 / 2}$, but both are comparable with an acceleration constant that appears ubiquitously in the dynamics of galactic systems.

Another observation that might indicate a connection of the local physics inherent in MOND with cosmology, is the aforementioned space conformal invariance of the deep-MOND limit of some NR versions of MOND. This invariance completes the space symmetry group of the theory to $\mathrm{SO}(4,1)$, which is the geometrical symmetry group of a de Sitter space-time [12]. 


\section{MOND vs CDM}

MOND and standard-dynamics-plus-DM are two competing paradigms now practiced side by side, with the adherents of each complacently pointing to the successes of their own favorite, and to the shortcomings of its competitor. The two are, however, not sister paradigms to be tested on the same touchstones. They account for the observed mass discrepancies in the universe in completely different ways, and judging them calls for different criteria. Take, for instance, the analysis of rotation curves by the two paradigms: It does not make good sense to judge with the same eye, and on equal footing, the agreement between the observed and theoretical curves for the two paradigms, since the MOND curves are inescapable predictions, while the DM theoretical curves are only fits, affording great latitude (usually with two parameters).

Consider, specifically, the now prevalent version of DM: the cold dark matter (CDM) paradigm. According to this picture, the mass discrepancies observed today in galactic systems are an outcome of the varied and turbulent histories of individual systems. Such histories involve the initial collapse of the system, possible mergers with other systems, violent collisions, gas accretion, dissipation, baryon heating and cooling, loss of matter from the system due to supernova explosions, AGN activity, and ram-pressure stripping, etc., etc. Under these processes, the dissipative and interactive baryons undergo very different influences from the presumably weakly interacting CDM. Thus the resulting relations between the distribution of the CDM and that of the baryons in a given systemwhich encapsulates the mass discrepancy-depend very strongly on the details of the history. These details are not known, and cannot be known, for a given individual system. CDM can thus not, generically, predict the mass discrepancy and its distribution, in an individual object (given the observed baryon distribution). One interesting and crucial exception to this concerns tidal dwarfs: the small "phoenix" galaxies that are born in gas tails produced in the aftermath of high-speed galaxy collisions. These dwarfs are formed in one, relatively clean, process, whereby previous history is erased. CDM predicts robustly that practically no DM finds its way into these dwarfs, contrary to what is claimed to be observed (see, e.g., [58, 59]). MOND does predict correctly the observed mass discrepancies in such systems.

If not predicting the details of the mass discrepancies in individual systems, can CDM predict, at least, general statistical correlations between DM and baryons in galactic systems, such as the different mass-velocity relations? These too would result from the above mentioned complicated, and little understood, physical processes, especially those involving the baryons. In CDM, attempts to predict such general correlations employ so called semi-analytic models. These purport to account for the complicated physical processes, putting in by hand all sorts of recipes, and formulas. But they are all based on guesses that, while educated, are quite unreliable. In any event, such considerations should necessarily lead to correlations with very large scattering, as the haphazard element cannot be avoided-while the observed correlations are known to be very tight.

What CDM can perhaps do well is predict statistical properties of pure CDM haloes-inasmuch as they are only little affected by the small amount of baryons now left in them. For example, the general statistics of halo population, and properties of the density distribution within a halo. It is well known, however, that the most straightforward predictions of this type (regarding, for example, the number of satellite halos, and the central density cusp predicted for CDM halos) are in conflict with observations; attempts to excuse such conflicts require high contortion skills. 
In contrast, in MOND, all aspects of the mass discrepancies are inescapable predictions, independent of the exact system history, and furthermore they all follow from the distribution of the baryons alone.

Epistemologically, the situation is similar to that concerning planetary systems, where we all agree on the underlying dynamics. Many of the properties of planetary systems at a given timesuch as the number of planets, their masses, compositions, and orbital radii-result from their complicated, and rather poorly understood, formation and evolution history. But other properties-such as those epitomized by Kepler's laws, and the dependence of Kepler's constant on the mass of the central star-are inescapable consequences of an undisputed, underlying theory of dynamics. We see in galaxies phenomenological laws that are as robust as Kepler's laws were, when they were recognized in the solar system, and which are universal. Does it make more sense to deduce that such regularities somehow resulted from a complicated and haphazard formation history of galaxies, or to accept that they are inevitable consequences of some appropriate underlying dynamics?

The main challenge that DM advocates now level at the MOND camp-as also echoed in this conference-is the remaining unproven ability of a MOND extension to account, as well as CDM does, for some cosmological observations-such as some aspects of the CMB power spectrum, and some aspects of large-scale structure. This is true: what standard dynamics has managed to explain in cosmology by invoking cosmological DM has not yet been shown to be fully accounted for by a relativistic MOND version (the effects of "dark energy" can be naturally accounted for in MOND, as I mentioned above in connection with BIMOND). TeVeS does go some way in this direction, but not fully, and BIMOND's implications on these points have not been explored enough, to say nothing of other possible MOND theories.

It has to be remembered, however, that what has already been accounted for by MOND in galaxies is much more than what still remains to be achieved in cosmology. In a sense, each galaxy is a universe of its own, offering as much data to be matched by a theory, as does cosmology at large. Newtonian dynamics was, after all, deduced with only one "mini-universe" in mind-the solar system. There is no reason to doubt that some relativistic version of MOND will be capable of accounting for the (epistemologically) little that still remains to be explained.

\section{References}

[1] M. Milgrom, Astrophys. J. 270, 365 (1983)

[2] R. H. Sanders, and S. S. McGaugh, An. Rev. Astron. Astrophys. 40, 263 (2002).

[3] J.D. Bekenstein, Contemp. Phys. 47, 387 (2006)

[4] M. Milgrom, In Proceedings XIX Rencontres de Blois; arXiv:0801.3133 (2008).

[5] M. Milgrom, In "The Invisible Universe International Conference", Paris, June 2009 (J.M. Alimi et al. eds.), arXiv:0912.2678

[6] R.H. Sanders, "The dark matter problem: a historical perspective", Cambridge U. Press (2010)

[7] M. Milgrom, Comm. Astrophys. J. 13, 215 (1989)

[8] M. Milgrom, Ann. Phys. 229, 384 (1994)

[9] R.H. Sanders, MNRAS, 296, 1009 (1998) 
[10] M. Milgrom, Phys. Lett. A 253, 273 (1999)

[11] L. Blanchet, and A. Le Tiec, Phys. Rev. D 80, 023524 (2009)

[12] M. Milgrom, Astrophys. J. 698, 1630 (2009)

[13] M. Milgrom, in Mass Profiles and Shapes of Cosmological Structures G.A. Mamon, F. Combes, C. Deffayet and B. Fort (eds) EAS Publications Series, 20, 217 (2006)

[14] J. Bekenstein and M. Milgrom, Astrophys. J. 286, 7 (1984)

[15] M. Milgrom, Phys. Rev. E 56, 1148 (1997)

[16] M. Milgrom, Mon. Not. R. Astron. Soc. 403, 886 (2010)

[17] J.D. Bekenstein, Phys. Rev. D 70, 083509 (2004)

[18] R.H. Sanders, Astrophys. J. 480, 492 (1997)

[19] C. Skordis, Class. Quant. Grav. 26, 143001 (2009)

[20] P.G. Ferreira, and G.D. Starkman, Science 326, 812 (2009)

[21] E. Sagi, Phys. Rev. D 80, 044032 (2009)

[22] S. Dodelson and M. Liguori, Phys. Rev. Lett. 97, 231301 (2006)

[23] C. Skordis, D.F. Mota, P.G. Ferreira, and C. Boehm, Phys. Rev. Lett. 96, 011301 (2006)

[24] C. Skordis, Phys. Rev. D 74, 103513 (2006)

[25] C. Skordis, Phys. Rev. D 77, 123502 (2008)

[26] T.G. Zlosnik, P.G. Ferreira, and G.D. Starkman, Phys. Rev. D 77, 084010, (2008)

[27] E. Sagi, Phys. Rev. D 81, 064031 (2010)

[28] T.G. Zlosnik, P.G. Ferreira, and G.D. Starkman, Phys. Rev. D 75044017 (2007)

[29] T. Jacobson and D. Mattingly, Phys. Rev. D 64, 024028 (2001)

[30] M. Milgrom, Phys. Rev. D 80, 123536 (2009)

[31] M. Milgrom, Mon. Not. R. Astron. Soc. 405, 1129 (2010)

[32] M. Milgrom, Phys. Rev. D 82, 043523 (2010)

[33] T. Clifton and T.G. Zlosnik, Phys. Rev. D 81, 103525 (2010)

[34] J-P. Bruneton, S. Liberati, L. Sindoni, and B. Famaey, JCAP, 03, 021 (2009)

[35] H.S. Zhao and B. Li, arXiv:0804.1588 (2008)

[36] X. Li and Z. Chang, arXiv:1005.1169 (2010)

[37] C.M. Ho, D. Minic, Y.J. Ng, arXiv:1005.3537 (2010)

[38] V. V. Kiselev and S. A. Timofeev, arXiv:1009.1301 (2010)

[39] J.M. Romero, R. Bernal-Jaquez, O. González-Gaxiola, Mod. Phys. Lett. A, 25 (29), 2501 (2010)

[40] S. S. McGaugh, preprint (2010)

[41] R. Scarpa, in "Structure Evolution and Cosmology", 2002, Santiago, Chile arXiv:astro-ph/0302445 (2003) 
[42] M. Milgrom, Mon. Not. R. Astron. Soc., 398, 1023 (2009)

[43] G. Gentile, B. Famaey, H. S. Zhao, and P. Salucci, Nature, 461, 627, (2009)

[44] F. Donato, et al., MNRAS, 397, 1169 (2009)

[45] M. Milgrom, Astrophys. J., 338, 121 (1989)

[46] M. Milgrom, Mon. Not. R. Astron. Soc., 326, 1261 (2001)

[47] R. H. Sanders, Third Aegean Summer School, “The Invisible Universe: Dark Matter and Dark Energy", arXiv:astro-ph/0601431 (2006)

[48] M. Milgrom, and R.H. Sanders, Astrophys. J., 599, L25 (2003)

[49] O. Tiret, F. Combes, G.W. Angus, B. Famaey, and H.S. Zhao, Astron. \& Astrophys., 476 L1 (2007)

[50] P.J. Humphrey, D.A. Buote, C.R. Canizares, A.C. Fabian, and J.M. Miller, arXiv:1010.6078 (2010)

[51] M. Milgrom, Astrophys. J., 577, L75 (2002)

[52] M. Milgrom, Astrophys. J., 478, 7 (1997)

[53] R.H. Sanders, Astrophys. J. 512 L23 (1999)

[54] E. Pointecouteau and J. Silk, Mon. Not. R. Astron. Soc., 364, 654 (2005)

[55] M. Milgrom, New Astron. Rev., 51, 906 (2008)

[56] R.H. Sanders, Mon. Not. R. Astron. Soc., 342, 901 (2003)

[57] G.W. Angus, Mon. Not. R. Astron. Soc., 394, 527 (2009)

[58] F. Bournaud, P.-A. Duc, E. Brinks, M. Boquien, M. Amram, U. Lisenfeld, B. S. Koribalski, F. Walter, and V. Charmandaris, Science 316, 1093 (2007)

[59] M. Metz, P. Kroupa, and H. Jerjen, Mon. Not. R. Astron. Soc. 394, 2223 (2009) 